\title{
A New Multi-Site Probe Array with Monolithically Integrated Parylene Flexible Cable for Neural Prostheses
}

\author{
Changlin Pang, Jorge G. Cham, Zoran Nenadic, Sam Musallam, Yu-Chong Tai, Joel W. Burdick, and \\ Richard A. Andersen
}

\begin{abstract}
This work presents a new multi-site probe array applied with parylene technology, used for neural prostheses to record high-level cognitive neural signals. Instead of inorganic materials (e.g. silicon dioxide, silicon nitride), the electrodes and conduction traces on probes are insulated by parylene, which is a polymer material with high electrical resistivity, mechanical flexibility, biocompatibility and easy deposition process. As a result, the probes exhibit better electrical and mechanical properties. The all dry process is demonstrated to fabricate these probe arrays with monolithically integrated parylene flexible cables using double-side-polished (DSP) wafers. With the parylene flexible cables, the probes can be easily assembled to a high density 3-D array for chronic implantation.
\end{abstract}

\section{INTRODUCTION}

$\mathrm{D}$ IRECT brain interfaces called neural prostheses are being designed that will enable a primate, via surgically implanted electrode arrays and associated computer algorithms, to control external electromechanical devices by pure thought alone (Fig. 1) [1-4]. The first beneficiaries of such technology are likely to be patients with spinal cord damage, peripheral nerve disease, or ALS (amyotrophic lateral sclerosis). The new multi-site neural recording probe array, described in this paper, is designed and used at the front end of the neural prostheses to record high-level cognitive signals.

Silicon has well-recognized advantages in probe fabrication. Currently, two main fabrication methods are developed to make silicon probes. One is based on boron diffusion and wet etching technologies [5], while the other depends on dry etching and silicon-on-insulator (SOI) technologies [6]. Each of these has its own unique set of

Manuscript received April 9, 2005. This work was supported by the National Institutes of Health

Changlin Pang is with the Caltech Micromachining Lab, California Institute of Technology, Pasadena, CA 91125 USA (phone: 626-395-2254; fax: 626-584-9104; e-mail: changlin@caltech.edu).

Jorge G. Cham is with the Department of Mechanical Engineering, California Institute of Technology, Pasadena, CA 91125 USA (e-mail: jgcham@caltech.edu).

Zoran Nenadic is with the Department of Mechanical Engineering, California Institute of Technology, Pasadena, CA 91125 USA.

Sam Musallam is with the Division of Biology, California Institute of Technology, Pasadena, CA 91125 USA.

Yu-Chong Tai is with the Caltech Micromachining Lab, California Institute of Technology, Pasadena, CA 91125 USA

Joel W. Burdick is with the Department of Mechanical Engineering, California Institute of Technology, Pasadena, CA 91125 USA.

Richard A. Andersen is with the Division of Biology, California Institute of Technology, Pasadena, CA 91125 USA. advantages and limitations. Most of these probes use silicon dioxide or silicon nitride as insulating materials. The rigidity of these materials results in large stresses. In addition, silicon dioxide is known to hydrate over time, which limits the probes' ability to be used for long time chronic neural recordings. Polymer materials are good substitutes. Parylene $\mathrm{C}$ was used as insulating layer on metal-wire neural probes by $\mathrm{Xu}$ [7], but a special process was required to open the probe tip, and had only a single electrode site per probe. Our work presented here first demonstrates the use of standard lithography processes to fabricate multi-site neural probes with parylene as insulating material.

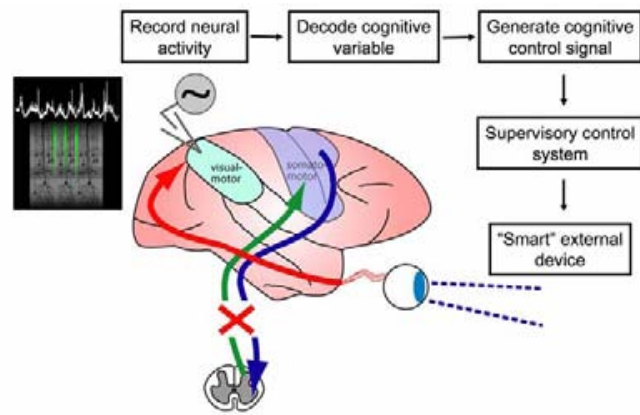

Fig. 1. Schematic of the pathway of information flow for the cognitive-based neural prosthetic paradigm. For visually guided movements, signals originating from the retinas pass through visual cortex and the posterior parietal cortex before arriving at motor cortex. Commands to move from motor and premotor areas converge to the spinal cord. In patients paralyzed by spinal cord damage there is a loss of motor signals down to the spinal cord, as well as of somatosensory feedback signals up to the motor and premotor areas. We propose to tap the early planning signals from the posterior parietal cortex. These cognitive signals will be decoded and used to generate control signals for supervisory control systems with hierarchical control of external devices.

Communication between the implanted probes to the outside word must be achieved via a multichannel interconnect cable. Even if telemetry is used, a cable is still necessary to link the probes to a telemetry platform. A lot of efforts were tried to solve this problem. One is the flexible printed circuit used by Norlin et al. [6]. But this cable can not be fabricated as small as the probe size, and a lot of wire bonding and epoxy gluing work is needed. One microfabricated cable is the silicon-based ribbon cable for the Michigan probes [5]. A shallow boron diffusion is used to define the cable substrate. The overall thickness of the cables is $4 \mu \mathrm{m}$, which makes the cables flexible. However, the authors report that the cables are not robust. Low yield is also reported for longer length cables because of the high aspect 
ratio. Obviously, polymer technology is a good substitute. We demonstrate an all dry process to fabricate parylene cables monolithically integrated with probe arrays. With the parylene flexible cables, the probes can be easily assembled to a high density 3-D array for chronic implantation.

\section{PARYLENE TECHNOLOGY}

Parylene is the generic name for members of a unique family of thermoplastic polymers that are deposited by using the dimer of para-xylyene (di-para-xylylene, or DPXN) [8]. As shown in Fig. 2, there are three different kinds of parylene commercially available on the market. Parylene $\mathrm{N}$ is poly-para-xylylene, a completely linear and highly crystalline polymer. Parylene $\mathrm{C}$ is basically parylene $\mathrm{N}$ with a chlorine atom replacing one of the aromatic hydrogens. Parylene D is similar to parylene $\mathrm{C}$ but with two aromatic hydrogens being replaced with chlorine atoms. The benzene backbone of the parylenes makes them very chemically inert, while the polyethylene-like interconnect makes it flexible.

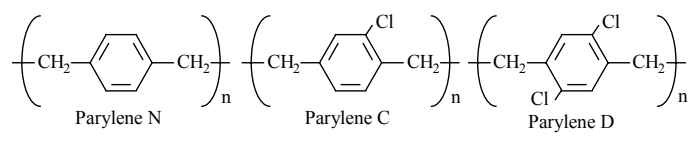

Fig. 2. The three most popular parylenes.

Table I gives some of the typical properties of parylene. Note that parylene has a high electrical resistivity and low Youngs modulus (2-5 GPa). With an elongation to break percentage of more than $200 \%$, parylene $\mathrm{C}$ is a perfect membrane material for large deflection applications.

Parylene is a biocompatible material. It is a proven USP (US Pharmacy) class-VI implantable plastic material.

Parylene is widely used for coating of biomedical devices (such as pacemakers and metal-wire neuroprobes). The main reason lies in its unique room-temperature CVD conformal deposition characteristics. Parylene is deposited at room temperature around 0.1 torr where the mean free path of the

TABLE I

TYPICAL PARYLENE PROPERTIES

\begin{tabular}{|l|c|c|c|}
\hline & $\begin{array}{c}\text { Parylene } \\
\mathrm{N}\end{array}$ & $\begin{array}{c}\text { Parylene } \\
\mathrm{C}\end{array}$ & $\begin{array}{c}\text { Parylene } \\
\mathrm{D}\end{array}$ \\
\hline Dielectric Strength $(\mathrm{V} / \mu \mathrm{m})$ & $>200$ & $>200$ & $>200$ \\
\hline Dielectric Constant & 2.6 & 3.1 & 2.8 \\
\hline Young's Modulus $(\mathrm{GPa})$ & $2-5$ & $2-5$ & $2-5$ \\
\hline Yield Strength $(\mathrm{MPa})$ & 42 & 55 & 62 \\
\hline Elongation to Break $(\%)$ & $20-250$ & 200 & 10 \\
\hline Density $\left(\mathrm{g} / \mathrm{cm}^{3}\right)$ & 1.12 & 1.29 & 1.42 \\
\hline Index of Refraction & 1.66 & 1.64 & 1.67 \\
\hline Melting Point $\left({ }^{\circ} \mathrm{C}\right)$ & 420 & 290 & 380 \\
\hline Glass Transition $\left({ }^{\circ} \mathrm{C}\right)$ & $>300$ & 240 & 240 \\
\hline Thermal Coef. of Expansion. $\left(/{ }^{\circ} \mathrm{C}\right)$ & $6.9 \times 10^{-5}$ & $3.8 \times 10^{-5}$ & $3-8 \times 10^{-5}$ \\
\hline Specific Heat at $20^{\circ} \mathrm{C}\left(\mathrm{cal} / \mathrm{g}^{\circ} \mathrm{C}\right)$ & 0.20 & 0.17 & - \\
\hline $\begin{array}{l}\text { Thermal Conductivity at } 20^{\circ} \mathrm{C} \\
\left(\text { cal } / \mathrm{cm}^{\circ} \mathrm{Cs}\right)\end{array}$ & $3.0 \times 10^{-4}$ & $2.0 \times 10^{-4}$ & - \\
\hline
\end{tabular}

molecules is on the order of $0.1 \mathrm{~cm}$. The first step of the deposition process is the vaporization of the solid dimer at approximately $150{ }^{\circ} \mathrm{C}$. The second step is the pyrolysis of the dimer at about $650{ }^{\circ} \mathrm{C}$, in which the dimer is cleaved at the two methylene-methylene bonds to yield the stable monomeric diradicals, para-xylylene. Finally, the monomers enter the deposition chamber at room temperature where they polymerize on the substrate. The cold trap at approximately $-70{ }^{\circ} \mathrm{C}$ is used to collect the un-reacted monomers before they enter the mechanical pump. Lift-off parylene skin technique is developed in our lab and is used to make parylene cables for the probe arrays.

\section{DEVICE DESIGN}

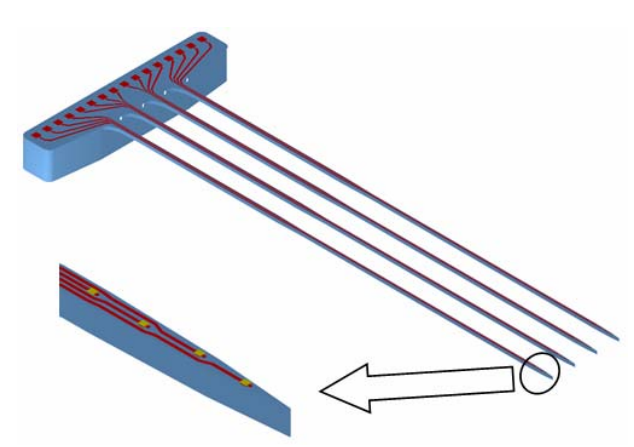

Fig. 3. Geometric design of the probes
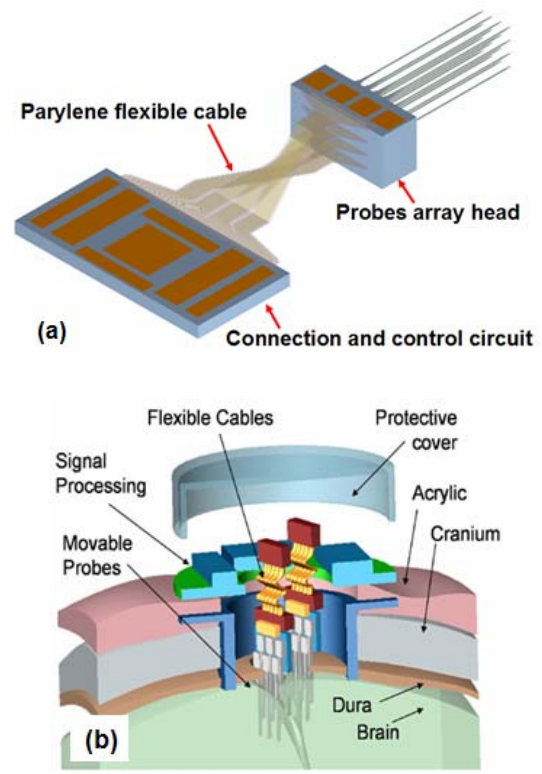

Fig. 4. (a) Schematic of a 3-D electrode array with parylene cables connected to connection and control circuit; (c) Schematic of cortical implantation using parylene cables.

The geometric design of the probes is shown in Fig. 3. Four shafts with four Pt electrode sites each protrude from a thicker plate. The target shaft thickness is $50 \mu \mathrm{m}$; the shaft width is $75 \mu \mathrm{m}$ at the bottom and $45 \mu \mathrm{m}$ at the outmost section. The lateral taper angle of the chisel-shaped tip is designed to $5^{\circ}$. 
The width of the trace lines at the outmost shaft section is 2 $\mu \mathrm{m}$. Different shaft length is designed for different testing purposes. $8 \mathrm{~mm}$ is for the acute recording of monkey neural signals, $4 \mathrm{~mm}$ and $2 \mathrm{~mm}$ long are for rats. The recording electrode sites are $10 \mu \mathrm{m} \times 10 \mu \mathrm{m}$ and are distributed with a $100 \mu \mathrm{m}$ pitch. The distance between the two shafts are 400 $\mu \mathrm{m}$, therefore, a 16-site 2-D electrode array is formed on one probe plate. Several plates can be stacked together to make a 3-D electrode array (Fig. 4(a)). We also designed $2 \mathrm{~mm}$ long probes with integrated parylene cables to do the chronic implantation recording. The parylene cables are monolithically integrated with the probes, and are basically a 'sandwich' structure, which has two parylene layers and metal traces between them. 16 parallel $\mathrm{Cr} / \mathrm{Au}$ trace lines are inside a parylene cable for the connection of the 16 electrodes on one probe plate. With the flexible parylene cables, we can easily implant the electrode array for chronic recording. Fig. 4(b) shows the whole design of implantation setup for chronic recording.

\section{FABRICATION PROCESS}

The silicon probe and parylene cable fabrication process flow is shown in Fig. 5, which is based on dry etching technology. Lift-off parylene skin technique is used to fabricate parylene cables monolithically. Instead of silicon-on-insulator (SOI) wafers, double-side-polished (DSP) wafers are used, which are less expensive. (a) XeF2 etching is performed on the probes top silicon surface to enhance the adhesion property between silicon and parylene [9]. (b) A photoresist sacrificial layer is patterned on the place where the parylene cables will be. (c) A $1.5 \mu \mathrm{m}$ parylene $\mathrm{C}$ insulating layer is conformably deposited. (d) $\mathrm{Cr} / \mathrm{Au}(\sim 100$ $\AA / 2000 \AA$ ) is thermal evaporated and patterned by lift-off to form the conduction traces. (e) A second parylene $\mathrm{C}$ layer $(\sim 1.5 \mu \mathrm{m})$ is deposited as a protective layer; the electrode sites and bonding pads are opened by plasma etching. (f) $\mathrm{Ti} / \mathrm{Pt}(\sim 200 \AA / 2000 \AA)$ is e-beam evaporated and patterned by lift-off to form the electrode sites. (g) The parylene layers are patterned along the probe shape by plasma etching. (h) Front side DRIE (deep reactive ion etching) ( 100 $\mu \mathrm{m}$ deep) is performed to define the probe shape into silicon. (i) Back side

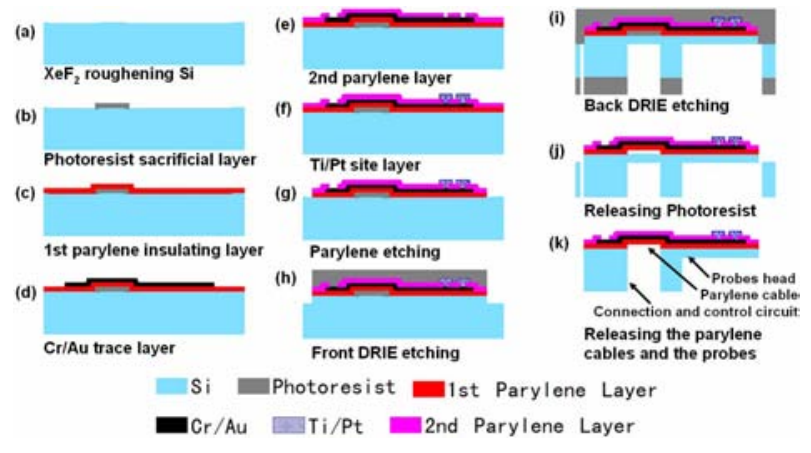

Fig. 5. Fabrication process flow.
DRIE defines the probe thickness, and releases the probes. During backside DRIE etching, the probes are protected by baked photoresist. By depositing protection photoresist on the backside of the finished probes die by die, the probe thickness can be well controlled in $5 \mu \mathrm{m}$ range on whole wafer. (j) Release all the mask and sacrificial photoresist. (k) Break the thin silicon film underneath the parylene cables to release them; break the connection parts between the wafer and the probe plates to release the whole devices.

\section{EXPERIMENTAL RESULTS}

Optical and SEM pictures of the fabricated probes are shown in Fig. 6. Parylene layer shows very good adhesion property on roughened silicon substrate. No visible delamination or adhesion defect is found between the first parylene layer and the probe silicon substrate, and between the two parylene layers. The probes were tested mechanically and electrically on rat cortex. The probes can be easily inserted into brain tissues without buckle or cracking. Electrode impedance is around $1.5 \mathrm{M} \Omega$ at $1 \mathrm{kHz}$. Neural signals were properly recorded. Fig. 7 shows the sample filtered neural data recorded from one channel of the neural probe in rat cortex; and the sample action potential waveforms.
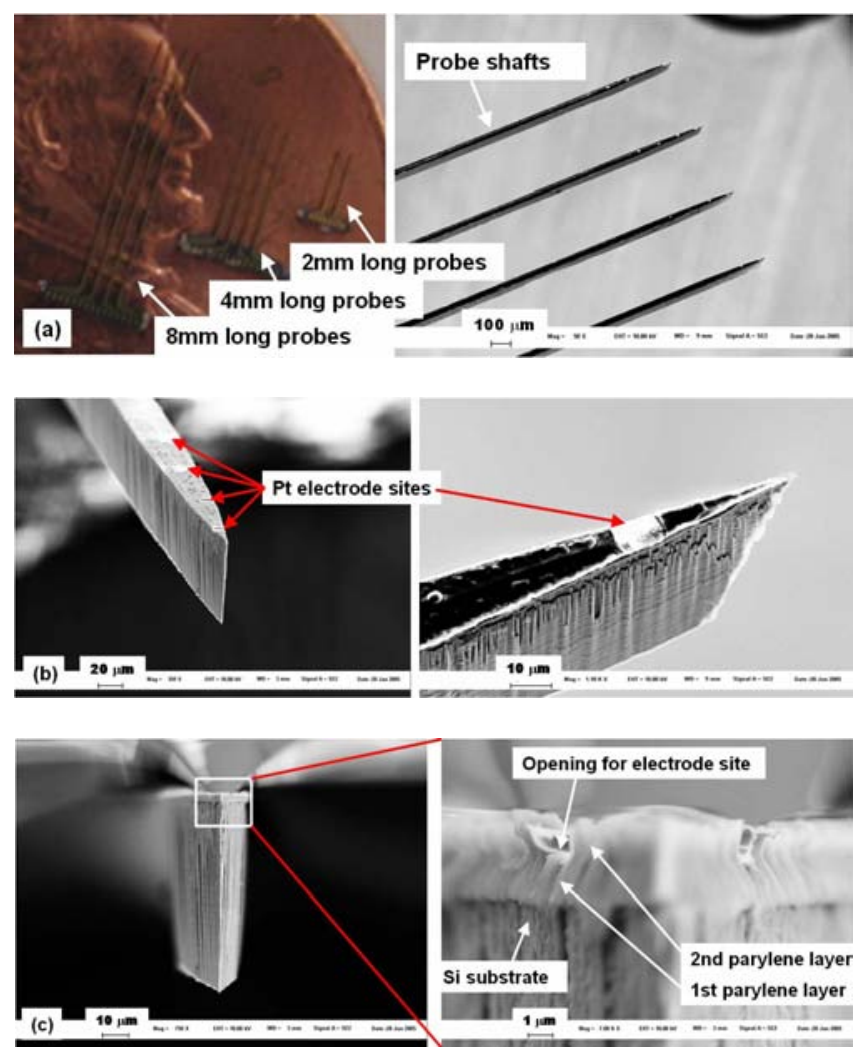

Fig. 6. Optical and SEM pictures of the fabricated probes. (a) Pictures of the whole probes and shafts; (b) SEM pictures of the probe tip with multiple electrode sites; (c) SEM pictures of front side view of the probe tip, showing interface between parylene layer and Si substrate and interface between two parylene layers. 
(a)

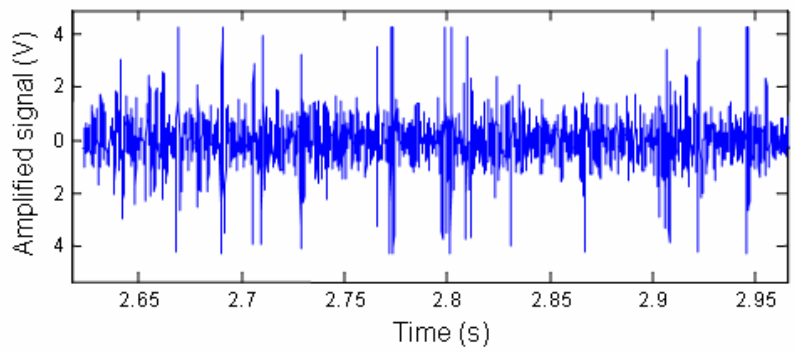

(b)

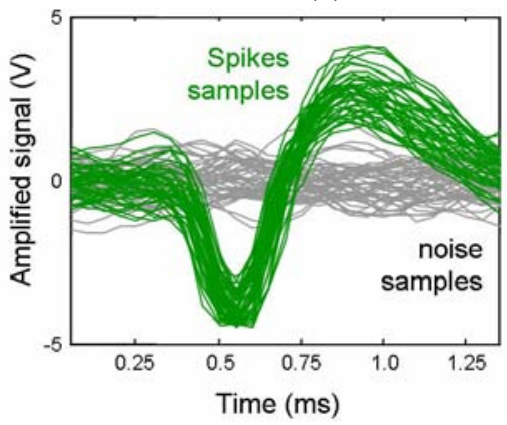

Figure 7: (a) Sample filtered neural data recorded from one channel of the neural probe in rat cortex; (b) Sample action potential waveforms.
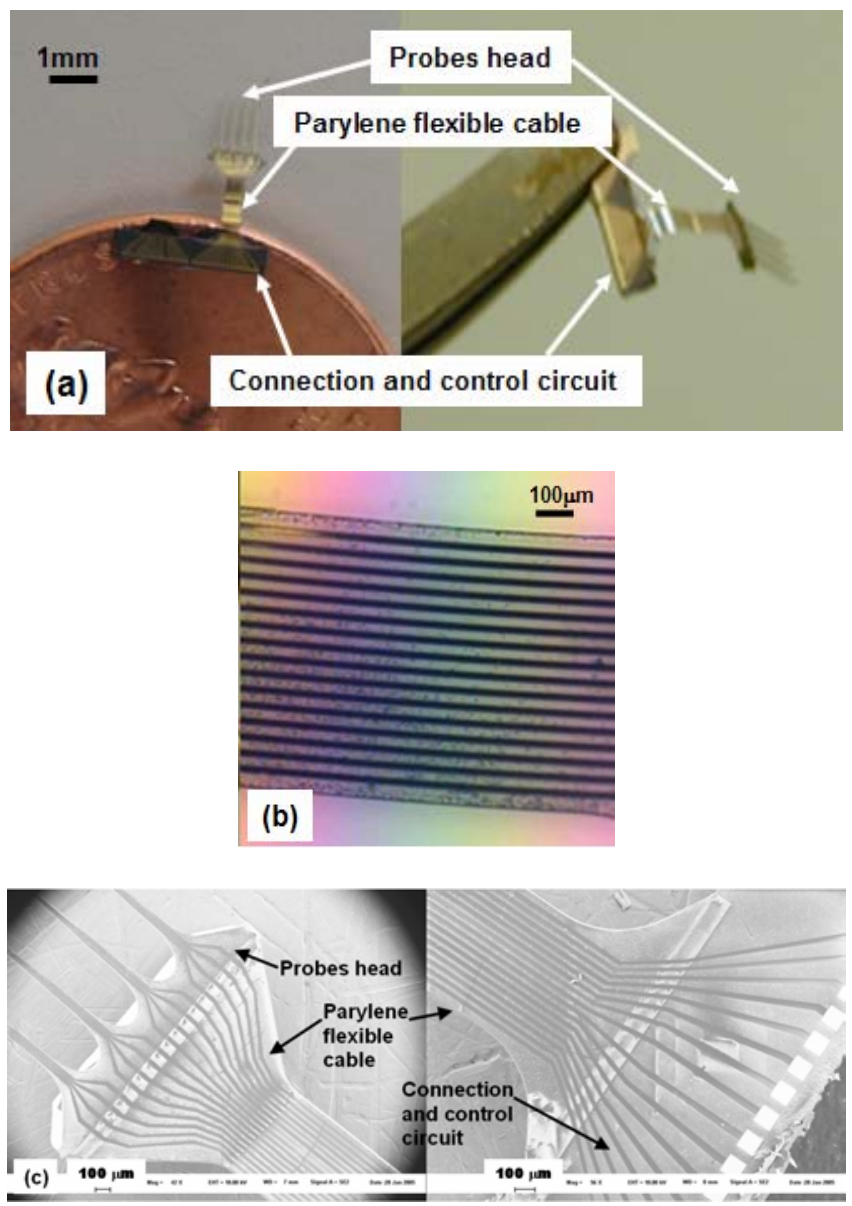

Fig. 8. Optical and SEM pictures of the fabricated parylene cables. (a) Optical pictures of the probe head and the connection circuit connected by parylene cable; (b) Optical zoom-in picture of the parylene cable; (c) SEM pictures of the probe head and the connection circuit connected by parylene cable.
The parylene cable is shown in Fig. 8. It is $3.2 \mathrm{~mm}$ long, $700 \mu \mathrm{m}$ wide and has 16 parallel $\mathrm{Cr} / \mathrm{Au}$ trace lines $(20 \mu \mathrm{m}$ wide) inside. Longer cables and more trace lines can be made using the same method. The parylene cable is monolithically fabricated with a probe array and a connection and control circuit part, and links the two parts together mechanically and electrically. The parylene cable is very flexible, you can even bend it to a sharp angle.

\section{CONCLUSION}

With good mechanical, electrical and biocompatible properties, parylene is an ideal material to be used as the insulating layer for the recoding probes and to make polymer cables for high density 3-D probe array used for chronic implantation. Experiments show good dielectric property of parylene $\mathrm{C}$ as insulating material on probes. The parylene process is easily compatible with the plasma etching process to make probes. An all dry process is demonstrated to fabricate the new multi-site probe arrays with monolithically integrated parylene cables. Neural signals were properly recorded from rat cortex.

\section{ACKNOWLEDGMENT}

We would like to thank Mr. Trevor Roper for assistance with fabrication and the members of the Anderson lab at Caltech for help on vivo testing.

\section{REFERENCES}

[1] S. Musallam, B. D. Corneil, B. Greger, H. Scherberger, and R.A. Andersen, "Cognitive Control Signals for Neural Prosthetics," Science, vol. 305 , issue 5681, pp. 258-262, 2004

[2] R.A. Andersen, J.W. Budrick, S. Musallam, B. Pesaran and J.G. Cham. "Cognitive Neural Prosthetics," Trends in Cognitive Sciences, vol. 8 , issue 11, pp. 486-493, 2004.

[3] Andersen, R.A., S. Musallam, B. Pesaran. "Selecting the Signals for a Brain-Machine Interface," Currrent Opinion in Neurobiology, vol. 14, pp. 1-7, 2004

[4] R.A. Andersen, J.W. Burdick, S. Musallam, H. Scherberger, B. Pesaran, D. Meeker, B.D. Corneil, I.Fineman, Z. Nenadic, E. Braunchaud, J.G. Cham, B. Greger, Y.C. Tai, and M.M. Mojarradi. "Recording Advances for Neural Prosthetics", Proceedings of the 26th Annual International Conference of the IEEE IMBS. San Francisco, CA, USA. Sepember 1-5, 2004, pp.5352-5355.

[5] K.D. Wise, D.J. Anderson, J. F. HETKE, D. R. KIPKE, AND K. NAJAFI, "Wireless Implantable Microsystems: High-Density Electronic Interfaces to the Nervous System," Proceedings of IEEE, vol. 92, no.1, pp.76 -97, 2004

[6] P. Norlin, M. Kindlundh, A. Mouroux, K, Yoshida and U. G. Hofmann, "A 32-site Neural Recording Probe Fabrication by DRIE of SOI Substrates," Journal of Micromechanics and Microengineering, vol.12, pp.414-419, 2002

[7] C. Xu, W. Lemon and C. Liu, "Design and Fabrication of A High Density Metal Microelectrode Array for Neural Recording," Sensors and Actuators, vol. A96, issue 1, pp. 78-85, 2002.

[8] M. Gazicki, G. Surendran, W. James, and H. Yasuda, "Polymerization of Para-Xylyene Derivatives (Parylene Polymerization). II. Heat Effects during Deposition of Parylene C at Different Temperatures", Journal of Polymer Science, vol. 23, pp. 2255-2277, 1985

[9] Q. He, C. Pang, Y.C. Tai and T. Lee, "Ion liquid chromatography on-a-chip with bead-packed parylene column," Proceedings, MEMS'04, Maastricht, the Netherlands, pp. 212-215, 2004 